\title{
What's on tablet PC: an introduction to FOAM at home
}

\author{
Adam J Singer ${ }^{1}$, Eric J Morley ${ }^{1}$, Haney Mallemat ${ }^{2}$ \\ ${ }^{1}$ Stony Brook University, Stony Brook, NY, USA \\ ${ }^{2}$ University of Maryland, Baltimore, MD, USA
}

Free open access medical education (FOAM) is a term broadly used to refer to medical education available at no cost. This education is made accessible through a variety of websites and online social media tools (e.g., Twitter, Google+, etc.). The term FOAM was created because there was much dissention from traditional academics when such education was associated with the term "social media". As a result, the term FOAM was created as a novel term without the negative connotation of social media. Today, the term FOAM encompasses more than simply online education; it also refers to a growing online community of educators and learners who also use the medium to disseminate and discuss medical information.

While some believe that FOAM is at odds with the more traditional research publication format we believe that these two important formats compliment each other and can live in harmony. This new form of accessing medical information has become especially popular with the younger generation of physicians and medical students and it is time that we embrace it and include in our family. In this issue we introduce a new section titled: "FOAM at home" in which we discuss information that we believe would be of interest to the readers of Clinical and Experimental Emergency Medicine. This section will be edited by Dr. Eric Morley, who will be soliciting contributions from experts in the field of FOAM.

Fee open medical education can take on a variety of formats (e.g., audio, text, etc.). Webposts on particular educational topics (i.e., blog posts) were the initial manifestations of FOAM. One of the most well recognized blog sites is LITFL (http://lifeinthefastlane.com). LITFL is one of the original educational blog sites known for emergency medicine and critical care and has now grown to be one of the premier online resources for clinicians. Medical education as in the form of pre-recorded audio (i.e., podcast) is another popular incarnation of FOAM. Podcasts have been around for over 10 years but have swiftly grown in the past few years and cater to diverse audiences ranging from medical student to senior clinicians. Educational videos are another popular version of FOAM; these videos can vary from a two-minute video on how to remove a ring (https: //www.youtube.com/watch?v=DxoAbK5Pc6w) or can be a full lecture that was recorded and then uploaded for viewing at a later time (https://vimeo.com/75337821). Websites like YouTube and Vimeo allow the free posting of videos and make the process relatively quick and simple.

The concept of FOAM also represents an online academic community where learners and educators can congregate online to discuss recent publications, debate controversial topics in medicine, or confer on difficult cases or encounters. These discussions typically occur over social media platforms with Twitter being most popular. This allows the FOAM community members to engage in discussion, network, and rapidly disseminate ideas. Furthermore, if information is inaccurate or misrepresented it can be vetted within the FOAM community.
eISSN: 2383-4625

Received: 10 June 2016

Revised: 14 June 2016

Accepted: 14 June 2016

Correspondence to: Haney Mallemat University of Maryland, 110 South Paca Street, 6th Floor, Suite 200, Baltimore, MD 21201, USA E-mail: Haney.Mallemat@gmail. com

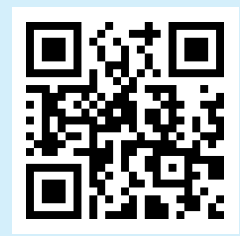

How to cite this article:

Singer $\mathrm{A}$, Morley $\mathrm{E}$, Mallemat $\mathrm{H}$. What's on tablet PC: an introduction to FOAM at home. Clin Exp Emerg Med 2016;3(3):181-182.

This is an Open Access article distributed under the terms of the Creative Commons Attribution Non-Commercial License (http:// creativecommons.org/licenses/by-nc/4.0/). 
〔EEM

Utilization and creation of FAOM does present some challenges. The end-user (the learner) is faced with countless on-line sources for FOAM. Selecting accurate and well-referenced material can be challenging. Services such as the ALiEM (https://www.aliem. com) approved instructional resources series have been established to provide curated content for learners. For creators of FOAM content the amount of work required to produce high quality material can be time consuming and it remains unclear how this will factor into academic advancement and promotion for many.

Future installments in the FOAM series will serve as a guide to the efficient use and creation of on-line material. We will explore different popular sites and apps first. We will then discuss using FOAM activities to build an educator portfolio and how to integrate this modality in residency and the medical school curriculum. The Explosion of FOAM in emergency medicine has been dramatic in recent years. It is critical we understand its pitfalls and full potential.

\section{CONFLICT OF INTEREST}

No potential conflict of interest relevant to this article was reported. 